\title{
ASSISTÊNCIA DE ENFERMAGEM PRESTADA A VÍTIMAS DE VIOLÊNCIA DOMÉSTICA EM UNIDADES DE PRONTO ATENDIMENTO
}

\section{NURSING ASSISTANCE PROVIDED TO VICTIMS OF DOMESTIC VIOLENCE IN EMERGENCY CARE UNITS}

\section{Maycon Hoffmann Cheffer ${ }^{1} *$ Caroline Mariana Valério $^{2} *$ Bianca Simardo Varella $^{3} *$ Bianca Machado Cruz Shibukawa ${ }^{4}$ Rafaela Bramatti Silva Razini Oliveira ${ }^{5} *$ Ariana Cristina Tasca $^{6} *$ Ieda Harumi Higarashi ${ }^{7}$}

\section{RESUMO}

Objetivo: descrever e identificar a percepção e atuação dos profissionais enfermeiros que trabalham em unidades de pronto atendimento, fazendo frente a assistência prestada a mulheres vítimas de violência doméstica. Métodos: estudo descritivo com abordagem qualitativa. Participaram do estudo 15 enfermeiros que atuam em duas Unidades de Pronto Atendimento no oeste do Paraná. Os participantes preencheram os questionários com perguntas acerca da assistência de enfermagem para vítimas de violência doméstica. Os dados passaram por análise temática de Minayo. Todos os preceitos éticos e legais foram cumpridos. Resultados: Foram identificadas cinco categorias: representando a violência doméstica pelo medo e sinais visíveis de agressão; aplicação do Protocolo de Manchester, acolhimento e encaminhamento à equipe multiprofissional; acolhimento e realização de curativos; atendimento multiprofissional humanizado e focado na resolução do problema; sentimento de impotência, despreparo e insegurança por parte da equipe e falta de assistência específica. Conclusões: o profissional enfermeiro é responsável por diversas etapas do atendimento as pacientes vítimas de violência doméstico, as quais vão desde a aplicação do Protocolo de Manchester, até mesmo a realização de curativos para as vítimas. Mesmo estes relatando sentimento de impotência e despreparo, os enfermeiros conseguem prestar uma assistência humanizada.

Palavras-chave: Equipe de Assistência ao Paciente; Violência Doméstica; Cuidados de Enfermagem; Papel do Profissional de Enfermagem.

\begin{abstract}
Objective: to describe and identify the perception and performance of professional nurses who work in emergency care units, facing the assistance provided to women victims of domestic violence. Methods: descriptive study with a qualitative approach. Fifteen nurses who work in two Emergency Care Units in western Paraná participated in the study. Participants filled out the questionnaires with questions about nursing care for victims of domestic violence. The data went through Minayo's thematic analysis. All ethical and legal precepts have been met. Results: Five categories were identified: representing domestic violence through fear and visible signs of aggression; application of the Manchester Protocol, reception and referral to the multiprofessional team; reception and placement of dressings; humanized multiprofessional service focused on solving the problem; feeling of helplessness, unpreparedness and insecurity on the part of the team and lack of specific assistance. Conclusions: the professional nurse is responsible for several stages of care for patients who are victims of domestic violence, ranging from the application of the Manchester Protocol, even performing dressings for victims. Even though they report feeling helpless and unprepared, nurses are able to provide humanized assistance.
\end{abstract}

Keywords: Patient Care Team; Domestic Violence; Nursing Care; Nurse's Role.

\footnotetext{
1 Doutorando em enfermagem - UEM. Docente de enfermagem do Centro Universitário Assis Gurgacz - FAG. E-mail: mayconcheffer@hotmail.com. ORCID: https://orcid.org/0000-0002-9361-0152

${ }^{2}$ Enfermeira. Centro Universitário Assis Gurgacz - FAG. E-mail: carolinemarianav @ hotmail.com. ORCID: https://orcid.org/0000-0002-2289-0054

${ }^{3}$ Discente de enfermagem do Centro Universitário Assis Gurgacz - FAG. E-mail: Bianca.simardo@ hotmail.com. ORCID: https://orcid.org/00000002-7393-0802

${ }^{4}$ Doutoranda em enfermagem no Programa de Pós-Graduação da Universidade Estadual de Maringá. Professora do departamento de enfermagem da Universidade Estadual de Maringá. E-mail: bih.cruuz@gmail.com. ORCID: https://orcid.org/0000-0002-7739-7881

${ }^{5}$ Doutorando em enfermagem - UEM. Docente de enfermagem do Centro Universitário Assis Gurgacz - FAG. E-mail: rafaelabramatti@ fag.edu.br. ORCID: https://orcid.org/0000-0003-1797-842X

${ }^{6}$ Docente de enfermagem do Centro Universitário Assis Gurgacz - FAG. E-mail: ariana@ fag.edu.br. ORCID: https://orcid.org/0000-0002-7062-3655 7 Professora doutora do Programa de Pós-Graduação de enfermagem. Universidade Estadual de Maringá, Maringá, PR, Brasil. E-mail: ieda1618@gmail.com. ORCID: https://orcid.org/0000-0002-4205-6841
} 


\section{INTRODUÇÃO}

A violência pode ser compreendida pelo uso de força física ou poder, sendo ela em forma de ameaças ou na prática, contra si próprio, outra pessoa, grupo e comunidade resultante em danos psicológicos, sofrimentos, algum tipo de privação, traumas ou morte ${ }^{(1)}$.

A violência doméstica, por muitas vezes sofrida pela mulher, é prevista pela Lei $\mathrm{n}^{\circ} 11.340$, popularmente conhecida por Lei Maria da Penha, a qual adota medidas para coibir e eliminar todas as formas de discriminação, violência doméstica e familiar contra a mulher, objetivando prevenir, punir e erradicar este tipo de violência ${ }^{(2)}$.

Segundo o Ministério da Saúde em dados disponíveis no Sistema de Informação de Agravos de Notificação, no ano de 2017 foram 220.559 casos de violência doméstica, sexual e/ou outras violências no Brasil, já no ano de 2018 esse número aumentou para 252. 668 novos $\operatorname{casos}^{(3)}$.

A China triplicou o número de denúncias de violência contra a mulher durante a pandemia do coronavírus. No ano de 2020, quando eclodiu a pandemia do COVID-19 no Brasil, houve aumento nos casos de violência doméstica contra a mulher. Este cenário se deve ao aumento do tempo de convivência com o parceiro, crise econômica e medo da pandemia ${ }^{(4)}$.

Desse modo, diante de uma vítima de violência doméstica a equipe de enfermagem tem o papel imprescindível de desempenhar as habilidades e competências necessárias para o seu acolhimento e tratamento, sempre promovendo a segurança do paciente e de sua família ${ }^{(4)}$

Considera-se importante que o profissional da saúde deva abordar o paciente de maneira acolhedora, respeitosa, segura, privativa, humanizada e preservando sua integralidade. Destaca-se, ainda, que o atendimento necessita de um cuidado contínuo, para que o processo ocorra o mais natural possível ${ }^{(1)}$.

Contudo, nem sempre os profissionais de saúde possuem treinamento específico para este tipo de abordagem ${ }^{(5)}$, o que ressalta a importância deste trabalho, tendo em vista que abordamos os sinais de agressões que são identificados pelos profissionais, a postura destes à frente dessa situação; como é realizado $\mathrm{o}$ atendimento humanizado $\mathrm{e}$ multiprofissional; e se estes receberam algum treinamento para realizar esse cuidado.

Nesse sentido, o estudo tem como objetivo descrever e identificar a percepção e atuação dos profissionais enfermeiros que trabalham em unidades de pronto atendimento, fazendo frente à assistência prestada a mulheres vítimas de violência doméstica.

\section{MÉTODOS}

Estudo exploratório e descritivo, com abordagem qualitativo. Pesquisas descritivas possuem como finalidade descrever com exatidão as características de determinado 
fenômeno. Nas pesquisas descritivas, o pesquisador registra e descreve os fatos observados sem interferi-los. Para coletá-los, utiliza-se de entrevistas, formulários, questionários, testes e observações, os quais permitem classificar, explicar e interpretar ${ }^{(6)}$.

Para tanto, foi realizada coleta de dados em um munícipio localizado na região Oeste do Paraná, com 328.454 habitantes ${ }^{(7)}$. Foram participantes do estudo 15 enfermeiros que atuam nas duas Unidades de Pronto Atendimento (UPA) do município, uma localizada na região norte e outra na região sul.

Os critérios para participar do estudo foram: ser enfermeiros das unidades de pronto atendimento e ter idade entre 21 e 60 anos. Como critério de exclusão, estabeleceu-se os profissionais que estivessem de férias, licenças, atestado ou que se recusasse a participar do estudo.

Devido ao cenário de pandemia do Covid-19 e isolamento social, optou-se pelo encaminhamento do formulário com perguntas objetivas e descritivas via Google Formulários com acesso à pesquisa por meio do grupo de aplicativo de conversa (WhatsApp).

O questionário de coleta de dados foi elaborado pelos pesquisadores após revisão da literatura e levantamento dos pontos mais importantes referentes à assistência de enfermagem, que o enfermeiro oferece à vítima de violência doméstica na unidade de pronto atendimento. Os dados foram coletados através de um guia de perguntas, contendo seis questões descritivas, para que os participantes respondessem como é sua atuação frente às vítimas de violência doméstica.

O formulário para a coleta de dados foi respondido entre os dias 13 e 24 de agosto de 2020, e permitiu caracterizar os participantes quanto a idade, sexo, tempo de serviço e experiência no atendimento de mulheres vítimas de violência doméstica.

As perguntas descritivas foram: 1) Como você identifica uma vítima suspeita de violência doméstica quando ela dá entrada na unidade de pronto atendimento?; 2) Quais foram suas condutas/encaminhamentos realizados para vítima?; 3) Quais os cuidados da assistência de enfermagem você realizou após a identificação?; 4) Como é o atendimento multiprofissional para as vítimas de violência doméstica?; 5) Você já participou de alguma formação/preparação para esse tipo de atendimento?; 6) Ao atender uma vítima de violência doméstica, qual a sua percepção em relação a assistência prestada?

As respostas foram transcritas na íntegra $\mathrm{e}$ as informações obtidas foram tratadas segundo a técnica da análise temática de Minayo realizada nas seguintes etapas: na pré análise foi realizada leitura flutuante e interpretativa do conjunto de dados, seguida de leitura extenuante para definição das unidades temáticas. $\mathrm{Na}$ exploração do material foi realizada a redução do texto e classificação e definição das 
unidades/categorias. No tratamento dos resultados obtidos e interpretação buscou-se a conferência dos dados obtidos com vista à concordância e solidez das categorias temáticas ${ }^{(8)}$.

Após análise e interpretação dos dados, emergiram cinco categorias, sendo elas: Identificando a violência doméstica pelo medo e sinais visíveis de agressão; Aplicação do Protocolo de Manchester, Acolhimento e encaminhamento à equipe multiprofissional; Acolhimento e realização de curativos; Atendimento multiprofissional humanizado e focado na resolução do problema e Sentimento de impotência, despreparo e insegurança por parte da equipe e falta de assistência específica.

As perguntas foram respondidas somente após os enfermeiros aceitarem o Termo de Consentimento Livre e Esclarecido (TCLE). O projeto de pesquisa foi aprovado pelo Comitê de Ética sob parecer $n^{\circ}$ 077406/2020, atendendo aos aspectos contidos na Resolução 466/2012 sobre pesquisa com seres humanos do Conselho Nacional de Saúde. O anonimato dos participantes foi garantido, substituindo seus nomes pelas iniciais e posteriormente identificados aleatoriamente pela sigla $\mathrm{E}$ seguido de um numeral.

\section{RESULTADOS}

Dos 15 participantes, $11 \quad(73,3 \%)$ pertenciam ao sexo feminino e quatro $(26,7 \%)$ ao sexo masculino. Referente à idade, três
(20\%) classificavam-se na idade entre 20-30 anos, oito $(53,3 \%)$ entre $30-40$ anos, $1(6,7 \%)$ entre 40-50 anos e três (20\%), entre 50-60 anos.

Com relação ao tempo de profissão, um entrevistado $(6,7 \%)$ possuía até cinco anos de profissão, cinco $(33,3 \%)$ de 5 a 10 anos, seis (40\%) de 10 a 20, dois (13.3\%) de 20 a 30 anos e um $(6,7 \%)$ com mais de 30 anos.

Todos os enfermeiros que participaram da pesquisa, descreveram já ter atendido vítimas de violência doméstica. Sobre a formação ou preparação para atendimento dessas, três (20\%) tiveram alguma preparação pela administração municipal, quatro $(26,6 \%)$ na graduação e oito $(55,3 \%)$ descreveram não ter nenhum preparo teórico.

\section{Identificando a violência doméstica pelo medo e sinais visíveis de agressão}

Os enfermeiros descreveram como identificam uma mulher com suspeita de violência. E as respostas coletadas foram: sinais da linguagem corporal como medo, pânico e também, a presença de ferimentos visíveis pelo corpo.

Vítima está sempre na defensiva, amedrontada (E2).

Geralmente apresentam marcas de agressão aparente, e seu discurso, na maior parte das vezes, tenta esconder a violência. O emocional delas sempre está muito abalado (E3). 
Quando chega apresentando sensação de pânico (E4).

Edema, hematomas e o próprio relato da mesma (E10).

Por seu primeiro relato, a maneira que se comporta e sinais visíveis de lesão" (E11).

Geralmente muito nervosa, sentindose ameaçada (E14).

Aplicação do Protocolo de Manchester, acolhimento e encaminhamento à equipe multiprofissional

No tocante às condutas que os enfermeiros realizaram após os atendimentos das vítimas de violência doméstica na unidade de pronto atendimento, as respostas foram: aplicação do Protocolo de Manchester, acolhimento e encaminhamento à equipe multiprofissional.

Protocolo de Manchester e assistente social (E1).

Acolhimento, inspirar confiança, notificar o caso; envolver multiprofissional (E2).

Classificamos conforme o Protocolo de Manchester, se o caso de enquadra em grave, tentamos conversar com o médico para priorizar o atendimento, evitando que a mesma fique exposta na recepção. Avisamos o serviço social para encaminhamentos posteriores $e$ notificação (E3).

Primeira ação é deixar a pessoa se sentir segura dando a ela além do tratamento físico, em caso de agressão, encaminhar para serviço social psicólogo e incluir na rede de proteção $(\mathrm{E} 4)$.
Acolhimento de enfermagem seguindo os itens da consulta de enfermagem (E6).

É priorizado o atendimento. Quando possível já colocado a paciente em local reservado. A triagem é realizada como prioridade no atendimento (amarelo ou laranja) (E9).

Conversa, atendimento preferencial, aconselhamento, contato com serviço social e psicológico para trabalho em conjunto (E14).

Acolhimento e realização de curativos

Ainda, sobre quais cuidados da assistência de enfermagem foram realizados para a mulher, após o enfermeiro identificar que se trata de uma vítima de violência doméstica, as respostas foram: acolhimento, cuidados com as lesões e a realização dos curativos necessários.

Preservação da identidade, minimizar a exposição, atuação ética, psicologia da enfermagem (E2).

Acolhimento, curativos, orientação nos cuidados com as lesões (E3).

Escuta ativa. Realização de curativos. Notificação de agressão. Comunicado polícia. E serviço social (E9).

Acolhimento $e$ atender da forma mais rápida e humanizada (E10).

Apoio emocional, cuidados com as lesões. Encaminhamento para serviços de apoio (E12).

Curativos, polícia e assistente social (E13).

Escuta qualificada, procedimentos curativos em caso de lesões, 
agendamento de procedimentos posteriores e acompanhamento geral (E14).

Atendimento multiprofissional humanizado e focado na resolução do problema

Ao descreverem como é o atendimento multiprofissional, as respostas foram: acompanhamento para o serviço social, encaminhamento ao psicólogo, atendimento humanizado e resolução do problema.

Ainda está fragmentado em alguns momentos da assistência. Requer melhor abordagem clínica e social com melhor preparo do multiprofissional para gerar um atendimento mais humanizado $e$ acolhedor (E2).

Focado na resolução do problema momentâneo e identificação de formas de continuidade do cuidado na alta desta paciente para que ela seja acompanhada e fortalecida pela rede de atenção à saúde do município com o objetivo de evitar que ela sofra nova violência (E3).

É muito importante porque cada profissional vai prestar seu atendimento específico dentro da sua área e com certeza a vítima será encorajada a tomar atitude certa diante do agressor que é a denúncia (E4).

Serviço social aborda a paciente. Buscando compreender a situação vivenciada para dar os encaminhamentos necessários (E9).

Se a agressão for física já é identificada pela equipe de enfermagem onde em conjunto com médico se trata o curativo primeiramente. Em todos os casos trabalha-se em conjunto com assistente social e acompanhamento psicológico" (E14).

Sentimento de impotência, despreparo e insegurança por parte da equipe e falta de assistência específica

Sobre as percepções, após a realização dos atendimentos prestados pelos enfermeiros, são relatados sentimento de impotência, despreparo, insegurança e a falta de profissionais para uma assistência específica, como a assistência social e psicológica.

Que a assistência ainda está fragmentada, mas de início visa curar os ferimentos e realizar os encaminhamentos (E1).

Que a paciente necessita do apoio humano do outro e que o profissional vá desprovido dos olhos do preconceito. Fará toda a diferença (E2).

De suma importância pois em alguns momentos o atendimento da enfermagem é o único apoio que a vítima possui (E4).

Que eu poderia ter feito mais, se a "rede" funcionasse na prática (E6).

Não existe muito preparo da equipe para cuidados com esses tipos de atendimento, apenas são encaminhados para serviços especializados, por isso não vejo muita perspectiva (E8).

Sentimento de Constrangimento. E as vezes impotência. Às vezes apatia. Quando os agentes da violência doméstica homem ou mulher estão etilizados (E9). 
A rede de proteção ainda não protege a mulher de maneira segura. Muitas vezes demora muito acolhimento (E12).

Que a escuta qualificada e o acolhimento em geral fazem parte da identificação da mesma. E que o olhar atento do profissional pode salvar uma vida vítima de violência e para isso os profissionais precisam estar preparados para olhar o paciente como um todo a sua frente (E14).

\section{DISCUSSÃO}

A violência está presente na sociedade desde o início da existência humana, independentemente da sua tipificação, tornando-se um problema historicamente mundial e de aspectos multifatoriais, principalmente associado ao gênero feminino.

O Brasil retém um alto número de vítimas, ocasionando uma situação a ser considerada um problema de saúde pública, consequentemente, gerando grandes custos para a nação ${ }^{(9)}$.

Quando a mulher se apresenta na unidade, o enfermeiro deve utilizar a anamnese para determinar fatores importantes, como seu círculo social, identificando pessoas próximas que podem colaborar, de alguma forma, com o tratamento, seja com apoio emocional, material ou serviços. O enfermeiro deve encorajar a mulher e orientá-la quanto a todas as opções de redes de assistência, utilizando de referenciais teóricos e metodológicos para aplicação de técnicas do atendimento ${ }^{(10)}$.
As mulheres apresentam sinais após a agressão, como insegurança, estresse, depressão, dificuldades de novos relacionamentos, dificuldades no sono, cefaleia, desconforto na coluna, náusea e hipertensão. Sinais que o profissional enfermeiro pode identificar durante a anamnese $^{(11)}$.

Os sinais que caracterizam comprometimentos na conservação de energia da mulher são os distúrbios do sono, desgaste físico, alimentação inadequada, fraqueza e falta de energia; características essenciais para integridade da mulher e que o enfermeiro deve se atentar. Ao exame físico, faz se necessário observar hematomas, escoriações, luxações e lacerações. No processo saúde e doença, observa-se obesidade, doenças imunológicas, gastrite e úlceras ${ }^{(12)}$.

A violência física deixa marcas aparentes no corpo, e transparecem efeitos negativos na saúde mental da mulher, principalmente pela baixa autoestima e a humilhação que elas sentem $^{(13)}$. As consequências da violência resultam em marcas físicas, psicológicas, sexuais, sociais e profissionais, o que faz necessário o encaminhamento para serviços de saúde especializados para enfrentar os problemas e ter uma melhora significativa na qualidade de $\operatorname{vida}^{(14)}$.

Algumas condutas do enfermeiro são necessárias para identificar que a mulher sofreu violência. Entre as consultas, o profissional precisa realizar anamnese com 
exame físico, verificação de sinais vitais, avaliação nutricional, padrão de eliminações e ciclos menstruais $^{(12)}$.

$\mathrm{O}$ profissional enfermeiro deve ficar à frente desse tipo de atendimento, sendo o primeiro contato e acolhendo a mulher. Posteriormente, deve prestar as devidas orientações e realizar os acompanhamentos e encaminhamentos a outros profissionais quando houver necessidade. Para isso, precisa estar capacitado e preparado para desenvolver tais habilidades e procurar capacitações em centros especializados ${ }^{(15)}$

Conforme também citado pelos enfermeiros no questionário, o Protocolo de Manchester é um grande aliado para determinar o tempo do atendimento da mulher e a complexidade, de acordo com o estado físico em que ela se encontra, diferenciando por cores os cinco níveis, separados pela queixa da mulher e visualização do enfermeiro $^{(16)}$.

Um dos importantes passos no atendimento é o acolhimento a vítima, de acordo com o Ministério da Saúde, é o ato ou efeito de acolher, uma ação de aproximação e uma atitude de inclusão. O acolhimento tem papel fundamental no momento em que as vítimas procuram atendimento. Nesse sentido, a assistência deve ser individualizada e humanizada, estabelecendo um vínculo afetivo e uma relação de empatia. O profissional deve mostrar à vítima que entende sua situação de dor e sofrimento ${ }^{(15)}$.
O cuidar das vítimas de violência doméstica exige mais do que somente habilidades técnicas, pois não há um modelo único a ser seguido. $\mathrm{O}$ cuidado requer uma atenção individualizada para tratar e curar, pois no momento em que a vítima procura um serviço de saúde, o profissional tem a oportunidade de acolhê-la e direcioná-la para os demais serviços ${ }^{(17)}$.

A qualificação da assistência prestada é favorecida, respeitando a privacidade dos usuários e deve se adequar ao ambiente e a cultura na qual eles se encontram. Ainda, o acolhimento desses pacientes, conforme a Política Nacional de Humanização (PNH) do Sistema Único de Saúde (SUS), deve conter as práticas e promoções de saúde ${ }^{(18)}$.

Dessa forma, para desenvolver o atendimento multiprofissional humanizado e focado na resolução do problema, o modelo de assistência deve ser intensificado no acolhimento do usuário. Cabe ressaltar, ainda, que junto com outras propostas de mudanças, como maior integralidade e universalidade no cuidado multiprofissional, visa o desenvolvimento da humanização do atendimento desses serviços ${ }^{(19)}$.

Os profissionais enfermeiros que atuam no atendimento às vítimas de violência doméstica relatam dificuldades na evolução do atendimento. Contexto esse que acontece, principalmente, pelas barreiras préestabelecidas pelas vítimas durante as ações prestadas. Tal fato se deve, geralmente, por causa de diversos fatores, como a 
insegurança, o medo, o constrangimento e a vergonha. Dessa forma, esses profissionais devem construir estratégias para se posicionarem como um facilitador do processo terapêutico, respeitando o contexto social e as particularidades de cada mulher, se aproximando da vítima e fornecendo o suporte necessário para as queixas ${ }^{(19)}$.

Ainda, as equipes relatam uma série de dificuldades nas ações prestadas, dentre elas, a fragmentação das instituições que fazem o atendimento dessas vítimas e a falta de colaboração, integração e compartilhamento de conhecimento entre os profissionais da área, o que gera obstáculos durante o atendimento. Entre os relatos obtidos, destaca-se o acolhimento individual realizado pelas equipes em diversos níveis multidisciplinares a principal forma para se garantir que o processo de atendimento seja concluído de maneira íntegra ${ }^{(19)}$.

Portanto, é notório, que a reunião das equipes, com participações de profissionais de diversas áreas do cuidado, como a da assistência social e da psicologia, aprimora e contribui com novas formas de se obter uma melhor definição das estratégias de cuidados a serem tomados, com o intuito de compartilhar responsabilidades e atribuições aos diversos setores do cuidado ${ }^{(20)}$.

\section{CONCLUSÃO}

O profissional enfermeiro é responsável por diversas etapas do atendimento às pacientes vítimas de violência doméstico, as quais vão desde a aplicação do Protocolo de Manchester, até mesmo a realização de curativos para as vítimas. Apesar dos relatos de sentimento de impotência e despreparo, os enfermeiros conseguem prestar uma assistência humanizada para estas mulheres.

No que diz respeito a problemática discutida, muitos conhecem a teoria, mas não sabem lidar com a situação e acabam não reagindo conforme o esperado, contudo, se moldam à necessidade da vítima e no sentido de resolubilidade, fazem o melhor dentro dos seus limites e dos limites impostos pelo sistema. Sendo assim, faz-se necessário mais estudos, pesquisas e investimentos em treinamentos sobre, caracterizada a importância e complexidade do assunto. Portanto, é primordial o entendimento amplo por parte do profissional para prestar um atendimento efetivo de forma individualizada no momento em que a vítima procura o serviço de saúde, pois é nesse momento que ela será direcionada aos demais encaminhamentos por parte do profissional.

\section{REFERÊNCIAS}

1. Machado LP, Freitag VL. Nursing care for a woman victim of sexual violence: a integrative literature review. RSD [Internet]. 2021 [citado 2021 fev 20]; 10(2): e33210212595. Disponível em: https://doi.org/10.33448/rsd-v10i2.12595

2. Brasil. Lei n. 11.340, de 07 de agosto de 2006. Cria mecanismos para coibir a violência doméstica e familiar contra a mulher, nos termos do $8^{\circ}$ do art. 226 da Constituição Federal, da Convenção sobre a Eliminação de 
Todas as Formas de Discriminação contra as Mulheres e da Convenção Interamericana para Prevenir, Punir e Erradicar a Violência contra a Mulher; dispõe sobre a criação dos Juizados de Violência Doméstica e Familiar contra a Mulher; altera o Código de Processo Penal, o Código Penal e a Lei de Execução Penal; e dá outras providências. Diário Oficial da União. De agosto de 2006. Disponível em: https://www2.camara.leg.br/legin/fed/lei/2006 /lei-11340-7-agosto-2006-545133normaatualizada-pl.pdf

3. Brasil, Ministério da Saúde. Banco de dados do Sistema Único de SaúdeDATASUS. Violência doméstica, sexual e/ou outras violências [citado 2021 fev 10]. Disponível em: http://tabnet.datasus.gov.br/cgi/tabcgi.exe?sin annet/cnv/violebr.def.

4. Vieira PR, Garcia LP, Maciel ELN. Isolamento social e o aumento da violência doméstica: o que isso nos revela? Rev Bras Epidemiol [Internet]. 2020 [citado $2021 \mathrm{fev}$ 15]; 23: E200033. Disponível em: https://doi.org/10.1590/1980-549720200033

5. Acosta DF, Gomes VLO, Oliveira DC, Gomes GC, Fonseca AD. Ethical and legal aspects in nursing care for victims of domestic violence. Texto Contexto Enferm [Internet]. 2017[citado 2021 fev 15]; 26(3): e6770015. Disponível em: https://doi.org/10.1590/010407072017006770015

6. Severino AJ. Metodologia do trabalho científico. $2^{\mathrm{a}}$ ed. São Paulo: Cortez; 2017.

7. Instituto Brasileiro de Geografia e Estatística (IBGE). População no último censo. Rio de Janeiro: IBGE; 2019 [citado 2021 jan 25]. Disponível em: https://cidades.ibge.gov.br/brasil/pr/cascavel/ panorama.

8. Minayo MCS, Deslandes SF, Gomes R. Pesquisa social: teoria, método e criatividade. Petrópolis: Vozes; 2016.

9. Lovi R, Hurley J, Hutchinson M. Qualitative findings from an Australian study of inter-partner violence content within undergraduate health curriculum.
Nurse educ. today. [Internet]. 2018 [citado 2021 fev 24]; 70: 1-7. Disponível em: https://doi.org/10.1016/j.nedt.2018.07.012

10. Arboit J, Padoin SMM, Vieira LB, Paula $\mathrm{CC}$, Costa $\mathrm{MC}$, Cortes LF. Atenção à saúde de mulheres em situação de violência: desarticulação dos profissionais em rede. Rev Esc Enferm USP [Internet]. 2017 [citado 2021 jan 20]; 5 (1):e03207. Disponível em: http://dx.doi.org/10.1590/S1980-

220X2016013603207

11. Spencer C, Mallory AB, Cafferky BM, Kimmes JG, Beck AR, Stith SM. Mental health factors and intimate partner violence perpetration and victimization: A metaanalysis. Psychol. Violence [Internet]. 2019 [citado 2021 fev 24]; 9(1): 1-17. Disponível em: https://doi.org/10.1037/vio0000156

12. Siqueira C, Rocha ES. Violência Psicológica contra a mulher: Uma análise bibliográfica sobre causa e consequência desse fenômeno. Revista Arquivos Científicos (IMMES) [Internet]. 2019 [citado $2021 \mathrm{fev}$ 24]; 2(1):12-3. Disponível em: https://doi.org/10.5935/2595-

4407/rac.immes.v2n1p12-23

13. Carneiro JB, Gomes NP, Estrela FM, Santana JD de, Mota RS, Erdmann AL. Violência conjugal: repercussões para mulheres e filhas(os). Esc. Anna Nery [Internet]. 2017 [citado $2021 \mathrm{Fev}$ 24]; 21(4): e20160346. Disponível em: http://dx.doi.org/10.1590/2177-9465-ean2016-0346.

14. Aragão FBA, Marinho $\mathrm{R}$ de $\mathrm{CO}$, Santos FBJ dos, Santos FS, Brandão LP, Aguiar JA, et al. Profile of women victims of sexual violence in Brazil: before and after the COVID -19 pandemic. RSD [Internet]. 2020 [citado 2021 Fev 24]; 9(10): e2289108114. Disponível em: https://doi.org/10.33448/rsdv9i10.8114

15. Rodrigues JB de S, Filha LR de L, da Costa YS, Santos J de S, da Silva MR, Brandão MA, et al. Atuação do enfermeiro frente a mulher vítima de violência sexual. REAS [Internet]. 2021 [citado 2021 fev 24]; 
13(2): 5801.

Disponível

em:

https://doi.org/10.25248/reas.e5801.2021

16. Roncalli AA, de Oliveira DN, Silva ICM, Brito RF, Viegas SMF. Manchester protocol and user population in the risk assessment: the nurse's view. Rev. baiana enferm [Internet]. 2017 [citado 2021 fev 24]; 31(2): e16949. Disponível em: : http://dx.doi.org/10.18471/rbe.v31i2.16949

17. Reis MJ, Lopes MHBM, Osis MJD. 'It's much worse than dying': the experiences of female victims of sexual violence. J Clin Nurs [Internet]. 2016 [citado $2021 \mathrm{fev}$ 24]; 26(15): 2353-61.

Disponível em: https://doi.org/10.1111/jocn.13247

18. Camargo DS, Castanheira ERL. Ampliando o acesso: o Acolhimento por Equipe como estratégia de gestão da demanda na Atenção Primária à Saúde (APS). Interface (Botucatu) [Internet]. 2020 [citado $2021 \mathrm{fev}$ 24]; 24(Supl. 1): e190600. Disponível em: https://doi.org/10.1590/Interface.190600
19. Trentin D, Vargas MAO, Lino MM, Leal SMC, Ferreira ML, Saioron I. Atendimento a mulheres em situação de violência sexual: revisão integrativa da literatura. Esc. Anna Nery [Internet]. 2019 [citado 2021 Fev 24]; 23(4): e20180324. Disponível em: https://doi.org/10.1590/2177-9465-ean2018-0324.

20. Moreira TNF, Martins CL, Feuerwerker LCM, Schraiber LB. A construção do cuidado: o atendimento às situações de violência doméstica por equipes de Saúde da Família. Saúde Soc. [Internet]. 2014 [citado 2021 fev 24]; 23 (3): 814-27. Disponível em: https://doi.org/10.1590/S010412902014000300007

Autor correspondente: Bianca Machado Cruz Shibukawa.

Endereço: Avenida Colombo, 5790. CEP: 87020-900. Bloco 2.

E-mail: bih.cruuz@gmail.com

Submissão: 2021-02-24

Aprovado: 2021-05-11 